\title{
Maximum length scale in density based topology optimization
}

\section{Lazarov, Boyan Stefanov; Wang, Fengwen}

\section{Published in:}

Computer Methods in Applied Mechanics and Engineering

Link to article, DOI:

10.1016/j.cma.2017.02.018

Publication date:

2017

Document Version

Peer reviewed version

Link back to DTU Orbit

Citation (APA):

Lazarov, B. S., \& Wang, F. (2017). Maximum length scale in density based topology optimization. Computer Methods in Applied Mechanics and Engineering, 318, 826-844. https://doi.org/10.1016/j.cma.2017.02.018

\section{General rights}

Copyright and moral rights for the publications made accessible in the public portal are retained by the authors and/or other copyright owners and it is a condition of accessing publications that users recognise and abide by the legal requirements associated with these rights.

- Users may download and print one copy of any publication from the public portal for the purpose of private study or research.

- You may not further distribute the material or use it for any profit-making activity or commercial gain

- You may freely distribute the URL identifying the publication in the public portal

If you believe that this document breaches copyright please contact us providing details, and we will remove access to the work immediately and investigate your claim 


\title{
Maximum length scale in density based topology optimization
}

\author{
Boyan S. Lazarov*, Fengwen Wang \\ Department of Mechanical Engineering, Solid Mechanics \\ Technical University of Denmark \\ Nils Koppels Allé, Building 404 \\ DK-2800, Denmark
}

\begin{abstract}
The focus of this work is on two new techniques for imposing maximum length scale in topology optimization. Restrictions on the maximum length scale provide designers with full control over the optimized structure and open possibilities to tailor the optimized design for broader range of manufacturing processes by fulfilling the associated technological constraints. One of the proposed methods is based on combination of several filters and builds on top of the classical density filtering which can be viewed as a low pass filter applied to the design parametrization. The main idea is to construct band pass filter which restricts the appearance of very thin and very thick elements in the design. In combination with the robust design optimization formulation the methodology results in manufacturable designs without the need of any post processing. The second technique provides more strict control on the maximum design features and is developed with the help of morphological operators. The formulation relies on a small number of additional constraints. Both approaches are demonstrated on optimization problems in linear elasticity.
\end{abstract}

Keywords: topology optimization, robust design, length scale, manufacturability

\section{Introduction}

The aim of this article is to present two new techniques for imposing maximum length scale in density based topology optimization. Topology optimization [1] is a design method which distributes material in a design domain by minimizing an objective function and fulfilling a set of constraints. The design is represented using a density field which takes value one at points occupied with solid material and zero in void regions. In order to utilize gradient based optimization techniques the optimization problem is relaxed and the density field is allowed to take values between zero and one. Existence of the solution is ensured by density filtering [2, 3]. Classical filtering techniques provide minimum length scale of the design, however, the regularized optimization process results in intermediate densities and practical realization of the final design requires post processing. Black and white (solid/void) designs can be obtained using projection techniques [4] with length scale imposed either on the solid or on the void phase. Alternatively

\footnotetext{
*Corresponding author

Email address: bsl@mek.dtu.dk (Boyan S. Lazarov)
} 
manufacturable black and white designs [5] can be obtained by requiring robustness of the performance with respect to geometric imperfections in the production process modeled by erosion and dilation operations [6].

The focus of most research works targeting length scale in topology optimization is on imposing minimum length scale and only few of them address the maximum length scale issue. The main advantages of imposing maximum length scale on optimized designs are defined clearly in [7]. Restrictions on the maximum length scale provide redundant members and diversification of the load path which results in more robust design with respect to localized damage [8]. The designers are provided with full control over the structure from technological perspective, e.g., avoiding additional effects not accounted for in the optimization formulation or the lack of production technologies for very thick elements. Maximum length scale is imposed in [7] by restricting the amount of material in the neighborhood of each point in the design domain which results in a large number of design constraints. Maximum length scale can be observed in the designs presented in [8], which is a result of requiring robust performance with respect to localized damage. The method is computationally expensive and is inapplicable in its current form for practical applications. Control on the maximum size of inclusions in periodic material designs by using combination of projections is demonstrated in [9, 10]. Algorithms for imposing maximum length scale in level set optimization approaches have been proposed recently in [11, 12]. An idea, similar to the one presented in [11], for controlling the minimum and the maximum length scale in optimized designs is proposed for the level set method in [13] and extended for density based topology optimization in [14]. The length scale control is achieved by restricting the density values for all skeleton points of the design. In both works [13, 14] the sensitivities related to changes of the skeleton are neglected and possible shortcomings are discussed in [12].

The current work presents two alternatives with the aim at eliminating the large number of constraints and reduce the computational cost. The gradients of the objective and the constraints are obtained using adjoint analysis and the chain rule without any simplifications. The first technique is based on the construction of a band pass filter in the frequency domain which restricts the appearance of thick members in the optimized design. The method does not introduce new constraints in the optimization formulation. The idea can be viewed as a generalization of the work presented in [15] where control of the structural complexity is demonstrated by sensitivity filtering of the low frequencies of the objective gradients. The second technique is based on morphological operators [16] and provides restrictions to the optimized design using one or two additional constraints. The constraints restrict the appearance of thick elements or large void regions. Both approaches can be arbitrary combined with any existing technique for imposing minimum length scale on the design, thus, providing the designer with full control over the geometric features of the optimized design. In addition an alternative scheme for filtering based on the Fast Fourier Transform is presented and discussed in details.

\section{Topology optimization}

As stated earlier topology optimization [1] is an iterative design method which distributes material in a given design domain $\Omega$ by minimizing an objective function (material volume, structural compliance, wave energy) and fulfilling a set of prescribed constraints. The design process is an iterative procedure and the design updates are based on the gradients of the objective and the constraints. The design domain is discretized using finite elements and the response of the associated physical problem is evaluated after each iterative design update step. The method has been applied in a broad range of optimization problems, e.g., heat transfer, linear elasticity, 
wave propagation [1]. Here the proposed maximum length scale techniques are demonstrated for optimization problems in linear elasticity. In discrete form the considered optimization problems can be written as

$$
\begin{aligned}
\min _{\rho} & : c(\boldsymbol{\rho}), \\
\text { s.t. } & : \mathbf{K}\left(\boldsymbol{\rho}_{d}(\boldsymbol{\rho})\right) \mathbf{u}=\mathbf{f}, \\
& : V\left(\boldsymbol{\rho}_{d}(\boldsymbol{\rho})\right) \leq V^{*}, \\
& : \rho_{\min } \leq \boldsymbol{\rho} \leq \rho_{\max }
\end{aligned}
$$

where $c(\rho)$ is the objective function. $\mathbf{K}\left(\boldsymbol{\rho}_{d}(\rho)\right) \mathbf{u}=\mathbf{f}$ is a discrete representation of the linear elastic problem, where $\mathbf{K}\left(\boldsymbol{\rho}_{d}(\boldsymbol{\rho})\right)$ is the so-called stiffness matrix obtained by a finite element discretization of the Navier-Cauchy partial differential equation. The continuous form of the optimization problem can be found in [1]. The nodal values of the displacement field are collected in the vector $\mathbf{u}$ and the external load supplied to the elastic system in the vector $\mathbf{f}$. The design field 1 is considered to be a constant within each element and all discrete values are collected in the vector $\rho$. The values for the first method, based on filtering, are required to be between $\rho_{\min }=-1$ and $\rho_{\max }=1$, which differs from the usual bounds $[0,1]$ utilized in most publications in topology optimization. For the second approach, based on morphological operators, the design field is between zero and one $0 \leq \rho \leq 1$. The density field $\rho_{d}, 0 \leq \rho_{d} \leq 1$, is obtained by series of transformations applied on the design field $\rho$. The transformations consist of several filtering, scaling and projections steps. All of them are discussed in details in the following sections. The total volume occupied with material is required to be smaller than a prescribed value $V\left(\rho_{d}(\rho)\right) \leq V^{*}$.

The material properties for each element $e$ are computed using the SIMP (Solid Isotropic Material with Penalization) interpolation scheme [17] given as

$$
E_{e}=E_{\min }+\rho_{d, e}^{p}\left(E_{\max }-E_{\min }\right)
$$

where $p$ penalizes the intermediate densities, $E_{\min }$ is a small value larger than zero which ensures positive definiteness of the stiffness matrix, and $E_{\max }$ is the elasticity modulus of the distributed material. The optimization problem is non-convex and the design can converge to a local minimum. The gradients of the objective with respect to the filtered variables are obtained by adjoint sensitivity analysis, and with respect to the design variables by employing the chain rule [1]. The objective is assumed to have the following general form $c(\boldsymbol{\rho})=\mathbf{l}^{\top} \mathbf{u}$. The sensitivities with respect to the density field variables $\rho_{d, e}, e=1, \ldots, N_{e}$ are given as

$$
\frac{\partial c}{\partial \rho_{d, e}}=-\lambda^{\top} \frac{\partial \mathbf{K}}{\partial \rho_{d, e}} \mathbf{u}=-\lambda_{e}^{\top} \frac{\partial \mathbf{K}_{e}}{\partial \rho_{d, e}} \mathbf{u}_{e}
$$

where $N_{e}$ is the number of elements in the mesh and $\lambda$ is the solution of the adjoint equation $\mathbf{K}\left(\rho_{d}(\rho)\right) \lambda=\mathbf{l}$. The vector $\mathbf{l}$ is equal to the external input of the system $\mathbf{l}=\mathbf{f}$ for minimum compliance problems. For mechanism design problems the vector $\mathbf{l}$ is used to select the displacement at the point of interest, therefore, $\mathbf{I}$ is zero everywhere except at the vector element corresponding to the selected degree of freedom.

\footnotetext{
${ }^{1}$ In this article the discrete representation of the density field $\rho$ will be interchanged with its continuous representation $\rho$ depending on the context.
} 


\section{Filtering in topology optimization}

The aim of the filtering step in the topology optimization is to ensure existence of the solution [2]. Designs obtained using the original topology optimization formulation, without any amendments, might result in several problems like checkerboards and mesh dependency. It is demonstrated in [18] and [19] that solutions consisting of alternating solid and void cells ordered in checkerboard-like patterns are not optimal and exist due to bad numerical modeling that overestimates the stiffness. Another issue is mesh dependency of the solution, i.e., refining the mesh and performing the optimization on the refined mesh might result in a completely different design rather than providing better description of the one obtained using the coarse mesh. Both of these problems are often avoided by applying filters on the original design field and modeling the physical density field to be equal to the result of the filtering process. The density field can be written as a convolution product of a filter function and the design field $\rho$

$$
\rho_{f}(\mathbf{x})=\int_{\Omega} F(\mathbf{x}-\mathbf{y}) \rho(\mathbf{y}) \mathrm{d} \mathbf{y}
$$

The filter function can be given in an explicit form as linearly or exponentially monotonically decaying function [3] or to be equivalent to the Green's functions of partial differential equation (PDE)[20]. In the second case the filtered field $\rho_{f}$ is obtained as the solution of the following PDE

$$
-r_{f}^{2} \nabla^{2} \rho_{f}(\mathbf{x})+\rho_{f}(\mathbf{x})=\rho(\mathbf{x}), \quad \mathbf{x} \in \Omega
$$

with prescribed boundary conditions. The parameter $r_{f}$ controls the minimum length scale in the design, and the prescribed boundary conditions control the density field along the boundary. A boundary condition of Neumann type $\frac{\partial \rho_{f}}{\partial \mathbf{n}}=0$ can be specified if the design problem does not require a prescribed density along the boundary. The Neumann boundary ensures volume preservation which simplifies the implementation of the volume constraint, i.e., the total volume of the design field is equal to the total volume of the filtered field.

\subsection{Interpretation of filtering in the frequency domain}

The filter Equation 4 can be viewed also as a low pass filter, where $\rho$ is an input signal to the linear system and $\rho_{f}$ is the filtered signal. The filter transfers the low frequency signals and attenuates the fast oscillatory part of the input. The filter properties can be analyzed using the so-called frequency transfer function, e.g. [21], which shows how the system reacts to every possible input frequency. The transfer function for the PDE filter in 1D can be written explicitly as

$$
H_{L}(\omega)=\frac{1}{r_{f}^{2} \omega^{2}+1}
$$

where $\omega$ is given angular frequency of the input signal and $H_{L}(\omega)$ denotes the frequency response function of the linear filter. A graph of $H_{L}(\omega)$ with respect to different angular frequencies is shown in Figure 1. As it can be seen the high frequencies are attenuated, i.e., the frequency content of the output signal consists of the low unattenuated harmonics of the input signal. Increasing the parameter $r_{f}$ attenuates faster the high frequency content of the input and decreasing it increases the high frequency content. In topology optimization, large parameter $r_{f}$ removes the fine details in the design and smaller $r_{f}$ provides finer details which can be described with more oscillatory harmonics. Therefore the minimum length scale is provided by suppressing the fine details (high frequencies) in the design field discretization. 


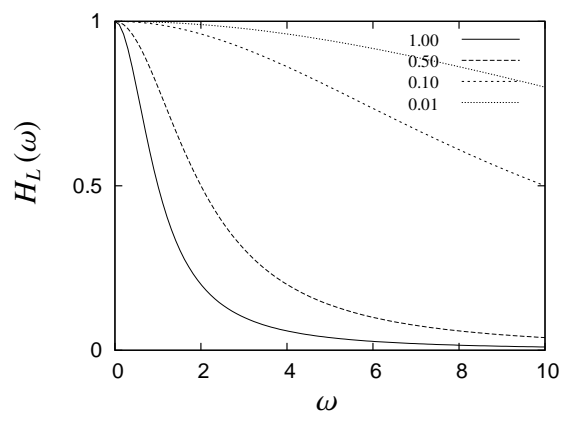

Figure 1: 1D low-pass filter with different parameters $r_{f}$.

\subsection{Band pass filter for topology optimization}

The low-pass filter includes all frequencies around the zero frequency and the maximum wave length of the harmonics which can contribute to the filtered design field is unlimited. Bounding it, provides maximum length scale in the design. Therefore, creating a band-pass filter provides bounds on both the minimum and the maximum length scale (wave length in 1D) of the filtered design field. One of the simplest operations which attenuates the response amplitudes around the zero frequency is the scaled Laplace operator $-r_{l}^{2} \nabla^{2}$. The frequency transfer function of the combined PDE filter and the Laplace operator is given as

$$
H_{H}(\omega)=\frac{r_{l}^{2} \omega^{2}}{r_{f}^{2} \omega^{2}+1}
$$

Taking the limit at $\omega \rightarrow \infty$ of Equation 7, reveals that the high frequency content of the design field is not attenuated. In topology optimization this results in fine design details comparable with mesh size, i.e., lack of minimum length scale. In order to attenuate the high frequency content, a second PDE filter with filter parameter $r_{s}$ can be introduced which results in the following frequency transfer function

$$
H_{B}(\omega)=\frac{r_{l}^{2} \omega^{2}}{\left(r_{f}^{2} \omega^{2}+1\right)\left(r_{s}^{2} \omega^{2}+1\right)}
$$

The parameters $r_{s}$ and $r_{f}$ can be used for tuning the frequency content of the filtered design and $r_{l}$ scales the amplitude of the response. The plots of Equation 7 and Equation 8 are shown in Figure 2. The maximum transmission of the filter defined by Equation 8 is located at $\omega=$ $\frac{1}{\sqrt{r_{s} r_{f}}}$ and the maximum is equal to $\frac{r_{l}^{2}}{\left(r_{f}+r_{s}\right)^{2}}$. In 2D and 3D the application of the above filtering steps results in pair or triplets of frequencies with weights found by rotating the 1D plot of the frequency transfer function around the center of the coordinate system.

Finite element implementation of the above filtering requires utilization of second or higher order elements for the numerical evaluation of the Laplace operator. However, close inspection of the equations reveals that the result from the operation can be obtained directly from Equation 5 by rearranging the terms as

$$
-r_{l}^{2} \nabla^{2} \rho_{f}(\mathbf{x})=\frac{r_{l}^{2}}{r_{f}^{2}}\left(\rho(\mathbf{x})-\rho_{f}(\mathbf{x})\right)
$$



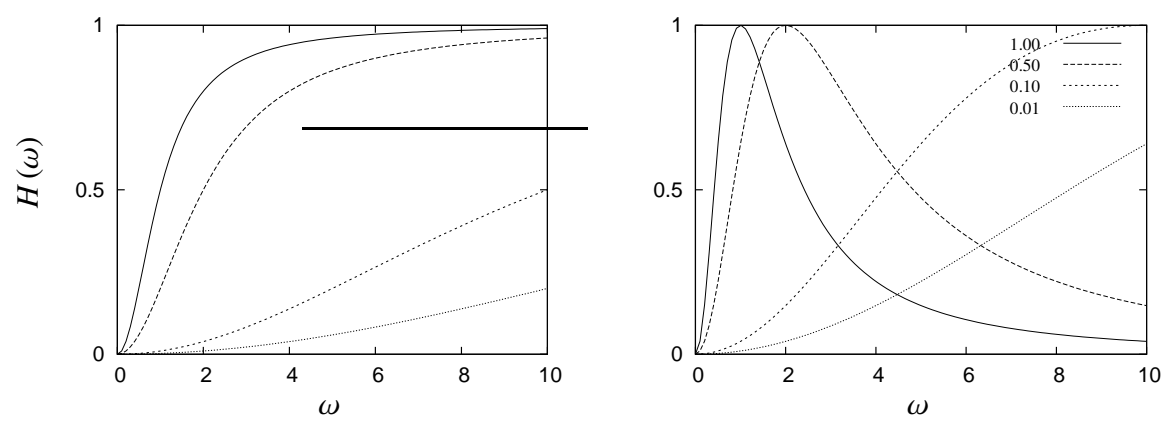

Figure 2: 1D high-pass (left) with $r_{f}=r_{l}$ and band-pass (right) filter with parameters $r_{f}=r_{s}$ and $r_{l}=2 r_{f}$.

The solution of the first filter with parameter $r_{f}$ is subtracted from the original density field, and after scaling is supplied as input to the second filter with parameter $r_{s}$, which is given as

$$
-r_{s}^{2} \nabla^{2} \rho_{s}(\mathbf{x})+\rho_{s}(\mathbf{x})=\frac{r_{l}^{2}}{r_{f}^{2}}\left(\rho(\mathbf{x})-\rho_{f}(\mathbf{x})\right)
$$

In order to utilize the result of the above band pass filter in topology optimization, the final density field needs to be bounded and the bounds need to be known in advance. The design fields $\rho(\mathbf{x})$ and $\rho_{f}(\mathbf{x})$ are bounded in the interval $[-1,1]$. The field $\rho_{s}(\mathbf{x})$ is bounded between the maximal and the minimal value of the field $\rho_{s}(\mathbf{x})$ which are controlled by the parameter $r_{l}$. An estimate for the maximal value of $\rho-\rho_{f}$ can be obtained by subtracting the upper and the lower bounds of $\rho$ and $\rho_{f}$, respectively. Utilizing this estimate for scaling the final density $\rho_{s}$ results in large gray regions and low contrast topologies. Sharper bounds can be obtained by using the Green's function of the filter. The filtered field $\rho_{s}$ is given as

$$
\rho_{s}(\mathbf{x})=\int_{\Omega} G(\mathbf{x}-\mathbf{y}) \rho(\mathbf{y}) \mathrm{d} \mathbf{y}
$$

where $G(\mathbf{x}-\mathbf{y})$ is the Green's function for the band pass filter, which for parameters $r_{f}=r_{s}=$ 1.0 and $r_{l}=2$ is shown in Figure 3 . In contrast to the standard filter where the filter function is strictly non-negative, the Green's functions for the band pass filter are positive close to the point $\mathbf{x}$ and become negative with increasing the distance $|\mathbf{x}-\mathbf{y}|$. The maximal value of $\rho_{s}$ can be obtained by setting $\rho(\mathbf{y})=-1, \forall \mathbf{y} \in\{\mathbf{y}: G(\mathbf{x}, \mathbf{y})<0\}$ and $\rho(\mathbf{y})=1, \forall \mathbf{y} \in\{\mathbf{y}: G(\mathbf{x}, \mathbf{y})>0\}$. Therefore the maximal value is evaluated as

$$
\rho_{s, \max }(\mathbf{x})=\int_{\Omega}|G(\mathbf{x}-\mathbf{y})| \mathrm{d} \mathbf{y}
$$

The output of the band pass filter can contain contributions of several frequencies with weight provided by the frequency response function. Therefore, the optimizer can utilize any of the harmonics with weights larger than zero. The interval can be narrowed by applying several bandpass filters one after another. Alternatively, the design field can be transformed using Fourier transform and the image can be convoluted with filter function defined entirely in the frequency domain. Such an approach will be discussed in details in the following section. 


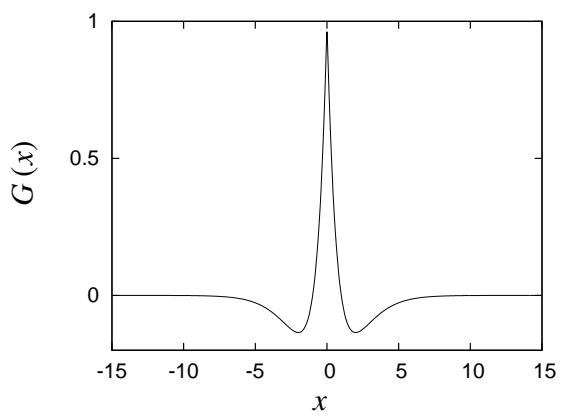

Figure 3: 1D Green's functions for parameters $r_{f}=r_{s}=1$ and $r_{l}=2$ and infinite filter domain.

Another important feature, of the PDE band-pass filter with Neumann boundary conditions, is that the integral of $\rho_{s}$ over the design domain is always equal to zero. Representing the density field $\rho_{d}$ using direct scaling of the filtered field $\rho_{s}$ with the inverse of the maximum and applying a shift of 0.5 would result in a design volume equal to 0.5 of the volume of the design domain. This limitation can be alleviated by using nonlinear scaling and shift different from 0.5 . In such cases the volume constraint in the optimization problem can become redundant.

The above filtering steps are also applicable to the classical density filtering techniques utilized in topology optimization, where the filter function has bounded support and is given as

$$
F(\mathbf{x}-\mathbf{y})=1-\frac{|\mathbf{x}-\mathbf{y}|}{R_{f}}, \quad \forall|\mathbf{x}-\mathbf{y}| \leq R_{f}
$$

A filtered design field $\rho_{f}$ with filter radius $R_{f}$ is subtracted from the original design field, and the result is provided as an input of a second filter with filter radius $R_{s}$. The frequency content of the obtained band pass filter can be computed numerically using the the Fast Fourier Transform (FFT).

\subsection{Band pass filter - FEM numerical implementation}

The band pass filter in discrete form can be written as

$$
\begin{aligned}
\mathbf{K}_{f} \boldsymbol{\rho}_{f} & =\mathbf{T}_{f} \boldsymbol{\rho} \\
\boldsymbol{\rho}_{o} & =\frac{r_{l}^{2}}{r_{f}^{2}}\left(\boldsymbol{\rho}-\boldsymbol{\rho}_{f}\right) \\
\mathbf{K}_{s} \boldsymbol{\rho}_{s} & =\mathbf{T}_{s} \boldsymbol{\rho}_{o}
\end{aligned}
$$

where $\mathbf{K}_{f}$ and $\mathbf{K}_{s}$ are obtained using finite element discretization of the filter operators Equation 5 and Equation 10, and projecting them onto the space of constant interpolation functions within each element (e.g. [22]), $\mathbf{T}_{f}$ and $\mathbf{T}_{s}$ are matrices which map the vectors with element-wise densities to nodal vectors, e.g. [20]. The vectors $\boldsymbol{\rho}, \boldsymbol{\rho}_{f}$ and $\boldsymbol{\rho}_{s}$ represent the original design field, the output of the first filter with length parameter $r_{f}$ and the output of the second filter. The final density is obtained by scaling and shifting the values of $\boldsymbol{\rho}_{s}$

$$
\rho_{d}=\frac{1}{2}\left(1+\frac{1}{M_{\rho_{s}}} \rho_{s}\right)
$$


a)

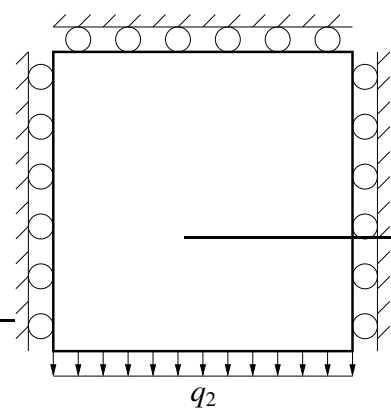

b)

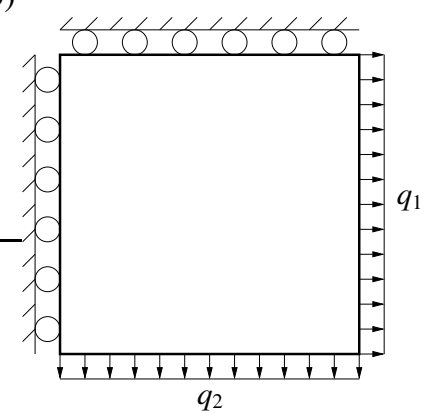

Figure 4: Design domain, load and boundary conditions for testing the max-length scale filter.

where $M_{\rho_{s}}$ is the maximal possible value of the filtered field $\rho_{s}$. The maximum for design domain without any bounds can be calculated using the analytic Green's functions of the PDE and Equation 12. For finite design domain the Green's functions depend on the prescribed boundary conditions and it is difficult to find exact analytic expression. Hence, for the discretized problems they can be computed numerically. The sensitivities of an element $\rho_{s, i}$ with respect to the original design field variables can be found using the chain rule. The filter operator is linear and hence, the vector with sensitivities $\mathbf{s}_{i}=\frac{\rho_{d, i}}{\rho}$ is a discrete representation of the Green's function associated with element $i$. Therefore, the maximal value of $\rho_{s, i}$ can be computed as

$$
M_{\rho_{s, i}}=\sum\left|\mathbf{s}_{i}\right|
$$

The maximal value within the whole design domain is given as $M_{\rho_{s}}=\max \left\{M_{\rho_{s, i}}, \forall i=1 \ldots N_{e}\right\}$. Such uniform scaling might introduce a bias in the design design domain, which might be undesirable. In such cases the scaling can be performed element wise with $M_{\rho_{s, i}}$. The field $\rho_{s, \max }(\mathbf{x})$ is smooth with main fluctuations close to the borders of the design domain. A numerical search procedure can be employed to decrease the cost of finding $M_{\rho_{s}}$. Another alternative is to use the fact that the arithmetic mean of positive variables is bounded from above by the quadratic mean, which can be utilized in finding analytic expression for upper bound of $M_{\rho_{s}}$. Both alternatives are left for future investigations.

The band-pass filter is demonstrated on two test cases shown in Figure 4. The results for vertical load (left case Figure 4) are shown in Figure 5 and the results for mixed loads are shown in Figure 6 . The penalty $p$ is set to $p=5$. The designs are obtained using the method of moving asymptotes (MMA) [23]. The domain dimensions are $25 \mathrm{~m} \times 25 \mathrm{~m}$ and the optimization is performed for 2D plane stress and unit thickness and unit modulus of elasticity.

The design shown in Figure 5 demonstrates clearly that the optimized topology has a frequency spectrum around the maximum transmission of the filter. For $r_{f}=r_{s}=0.5$ the maximum transmission is at $\omega=2$. The corresponding wavelength is $\pi$, which for the length of the design domain results in 7.95 waves. The estimate is slightly larger than the obtained one. The number of bars estimated by visual inspection is equal to 7 . It should be pointed out that the uniqueness of the topology cannot be guaranteed. For different initial topologies and iteration histories the designs differ, however, in all cases the number of the vertical bars is around the estimated number of 7.95. For the case $r_{f}=r_{s}=1.0$ the estimated number of bars is 3.97 which is very close to the obtained number of bars estimated by visual inspection and equal to 4 . Similar behavior 


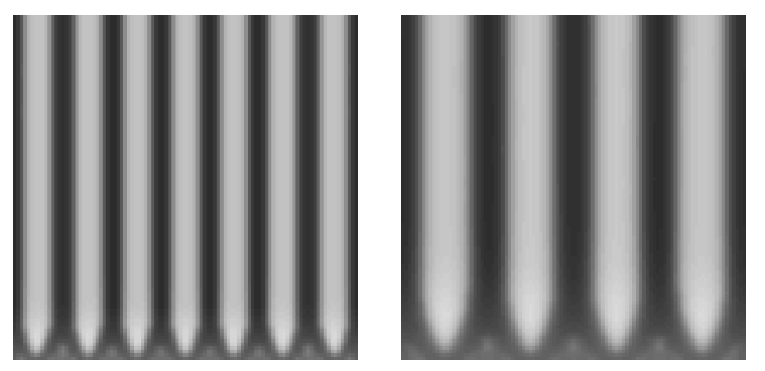

Figure 5: Topology optimized design for $q_{2}=0.01 \mathrm{~N}$ and parameters $r_{f}=r_{s}=0.5$ (left), and $r_{f}=r_{s}=1.0$ (right) Boundary conditions and the load is shown in Figure 4 a).
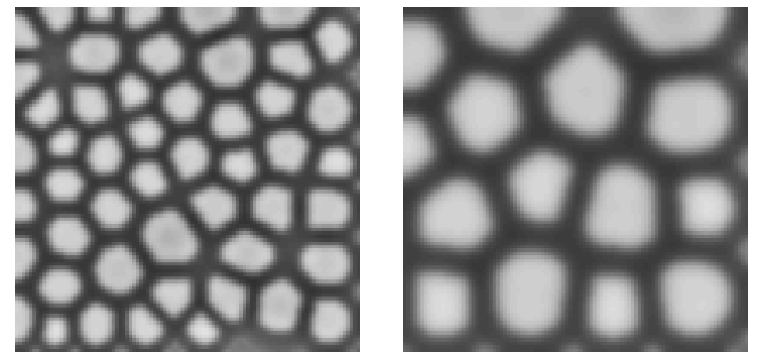

Figure 6: Topology optimized design for $q_{1}=q_{2}=0.01 \mathrm{~N}$ and parameters $r_{f}=r_{s}=0.5$ (left), and $r_{f}=r_{s}=1.0$ (right). Boundary conditions and the load is shown in Figure 4 b). 
can be observed for the mixed case results shown in Figure 6 A common feature for all of the presented designs is the lack of contrast. The maximal value $M_{\rho_{s}}$ can be achieved only by a single pattern of design distribution which usually does not coincide with the optimal solution of the problem. The obtained densities $\rho_{d}$ are far from the zero/one bounds and stay closer to 0.5 . In this case, the optimizer can have difficulties finding optimal design even for high penalization factor $p$. A possible solution is to use point wise nonlinear transformation(projection) which compresses the design space around 0 and 1 . Further details are discussed in section 4

\subsection{Band-pass FFT filter}

More precise control over the frequency transfer function of a band-pass filter can be achieved by modeling it directly in the frequency domain, rather than obtaining it through Fourier transform of some analytic or PDE spatial filter. Often analytic representation of such filters cannot be found in the physical domain. Therefore, topology optimization applications can utilize numerical implementations based on FFT. The design field $\rho$ is transferred in the frequency domain $\tilde{\rho}(\omega)=\mathcal{F} \rho(\mathbf{x})$ using $1 \mathrm{D}, 2 \mathrm{D}$ or $3 \mathrm{D}$ Fourier transform and the corresponding FFT implementation. In the frequency domain $\tilde{\rho}(\omega)$ is convoluted using frequency transfer function defined entirely in the frequency domain and the result is converted back in the spatial domain using the inverse Fourier transform. The above operations can be written as

$$
\begin{aligned}
\tilde{\boldsymbol{\rho}}(\boldsymbol{\omega}) & =\mathcal{F}(\boldsymbol{\rho}(\mathbf{x})) \\
\tilde{\boldsymbol{\rho}}_{s}(\boldsymbol{\omega}) & =\tilde{F}(\boldsymbol{\omega}) \tilde{\boldsymbol{\rho}}(\boldsymbol{\omega}) \\
\boldsymbol{\rho}_{s}(\mathbf{x}) & =\mathcal{F}_{i}\left(\tilde{\boldsymbol{\rho}}_{s}(\omega)\right)
\end{aligned}
$$

where $\mathcal{F}_{i}$ denotes the inverse of the transformation and $\tilde{F}(\omega)$ denotes the filter function defined entirely in the frequency domain. The Fourier transform is infinitely periodic in all dimensions. In order to decrease the appearance of artifacts around the edges of the design domain, the discretized design field can be padded with zeros. After filtering the result is cut back to the original size. In a similar way the boundary condition of the design can be modified by padding the design domain with predefined patterns different than zero. In numerical implementations the Fourier and the inverse Fourier transforms are replaced with FFT and iFFT.

The gradients of any function $\mathbf{s}=\frac{\partial c\left(\rho_{s}(\rho)\right)}{\partial \rho}$ with respect to the original design variables can be obtained by knowing the gradients $\mathbf{s}_{s}=\frac{\partial c\left(\rho_{s}\right)}{\partial \rho_{s}}$ and passing them through the filter

$$
\begin{aligned}
\tilde{\mathbf{s}}_{s} & =\operatorname{FFT}\left(\mathbf{s}_{s}\right) \\
\tilde{\mathbf{s}} & =\tilde{\mathrm{F}} * \tilde{\mathbf{s}}_{s} \\
\mathbf{s} & =\operatorname{iFFT}\left(\tilde{\mathbf{s}}_{\mathbf{s}}\right)
\end{aligned}
$$

The maximum value $M_{\rho_{s, i}}=\sum\left|\mathbf{s}_{i}\right|$ can be found by passing vector $\mathbf{s}_{s}$ through the filter which consists of zeros for all elements with index $j \neq i$, and one at position $i$.

A band-pass filter in the frequency domain is modeled by a donut like filter function in the frequency domain. The transformed filter function in the physical domain is shown in Figure 7 The filter function in the frequency domain is defined as

$$
F(\omega)=\exp \left(-\frac{\left(|\omega|-\omega_{s}\right)^{2}}{2 s^{2}}\right)
$$




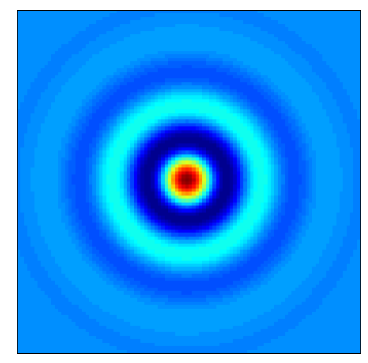

Figure 7: Spatial filter function for the central frequency $\omega_{s}=0.05$ cycles/element and deviation parameter $s=0.0075$.

where $|\omega|$ is the length of the vector $\omega$. The bandwidth of the filter is controlled by the parameter $s$, and the central frequency is given by $\omega_{s}$. Designs for settings shown in Figure 4 are similar to the results from the PDE based implementation, shown in Figure 5 and Figure 6, and therefore, they are not presented here. However, the band-pass FFT filter is utilized in the examples presented later in this work.

It should be pointed out that the traditional low-pass filter utilized for imposing minimum length scale in topology optimization can be implemented using FFT where the donut is replaced by a ball with center around zero. FFT low- and band-pass implementations based on highly tuned numerical FFT libraries can potentially outperform the PDE filters and provide effective filter solution in topology optimization independent of the length scale imposed by the filter. A possible limitation of the filter is that it is applicable to regular rectangular domains only. Irregular domains can be extended to rectangular ones by padding the design field with zeros, which can increase the computational cost associated with the filter.

\section{Black and white designs based on band-pass filters}

Optimized designs where the physical densities coincide with the filtered fields possess large gray regions and practical realizations require post-processing. The post-processing step might completely destroy the design performance $[6,24]$. Thus, several schemes to alleviate it have been proposed in the literature [4, 25, 26, 27]. The main idea is to project the filtered field above given threshold to one and below the threshold to zero. The projection is embedded in the topology optimization algorithm and the threshold projection does not affect the optimality of the solution. Mathematically the projection is expressed using Heaviside function. Since the Heaviside function is not differentiable, it is replaced with regularized approximation, e.g. $[4,25,26,27,6]$. In this section the projected field is represented as

$$
\rho_{d}=\frac{1}{2}\left[1+\tanh \left(\beta\left(\rho_{s}-\eta\right)\right)\right]
$$

where the parameter $\beta$ controls the quality of the approximation and $\eta$ is provided threshold. As $\beta \rightarrow \infty$, the tanh function approaches the Heaviside function. To provide exact $0 / 1$ bounds for the minimum and the maximum of $\rho_{d}$ the above expression can be normalized [6], however, for simplicity the normalization is avoided here.

Three designs obtained using threshold projections, with threshold $\eta=0$, are shown in Figure 8 The left design is obtained with uniform initial guess. The topology resembles closely 


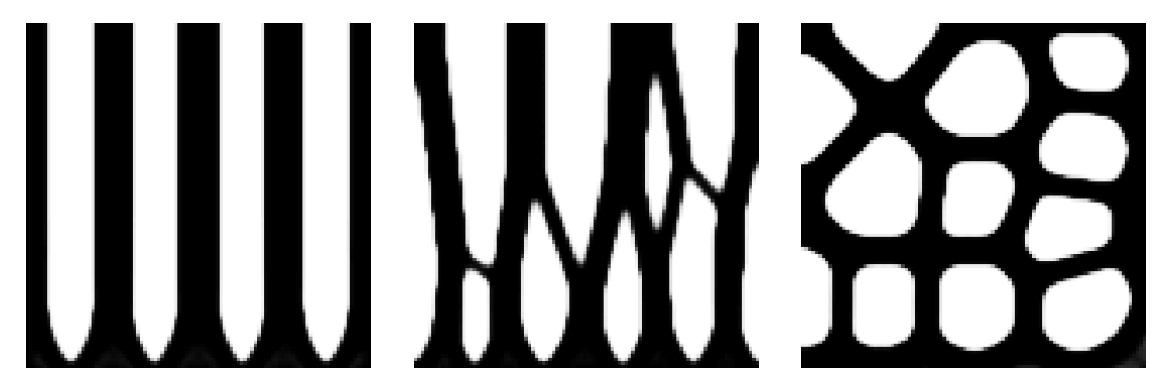

Figure 8: Topology optimized design for parameters $r_{f}=r_{s}=1.0, \beta=25$ and, $q_{2}=0.01 \mathrm{~N}$ uniform initial guess (left), $q_{2}=0.01 \mathrm{~N}$ random initial guess (middle), and $q_{1}=q_{2}=0.01 \mathrm{~N}$ random initial guess (right). Boundary conditions and the loads are shown in Figure 4 a) for the left and the middle designs and Figure 4 b) for the right design.

the topology of the design obtained without projection and shown in Figure 5, Designs with projection possess much better contrast and they are close to discrete $0 / 1$ designs. In order to obtain sharp transition between the void and solid regions the projection amplifies the high frequencies in the design spectrum. Therefore, the minimum length scale introduced by the filtering process is lost. The effect can be observed on Figure 8 for the design obtained with random initial guest.

It is demonstrated in [28, 6] that the appearance of small features in the design can be suppressed by replacing the objective in the original topology optimization formulation given by Equation 1 with the worst objective obtained for three thresholds $\eta_{i}, \eta_{d}$ and $\eta_{e}$ representing the blueprint, the most dilated and the most eroded designs, respectively. More realistic imperfections can be obtained by varying $\eta$ in a prescribed interval $\left[\eta_{d}, \eta_{e}\right]$ and modeling the threshold as random variable. The model can represent uniform production errors due to erosion or dilation along the perimeter of the design [29]. The optimization formulation has been extended later to include spatial variations of the geometry along the design perimeter [30, 31], and recently, it has been demonstrated that the threshold projection schemes resemble closely micro/nanolithography and photolitography production processes [32, 33]. The development opens new possibilities for obtaining more realistic physical models of the designs and incorporating them in the optimization process. Requiring robustness of the design, i.e., lack of sensitivity of the objective with respect to variations in the geometry, often leads to black and white design with clearly defined minimum length scale. It should be pointed out that the length scale is defined only when all design realizations share the same topology. In all cases where the design topology changes between the realizations, the removal or the addition of elements to the design due to variations in the manufacturing process does not change significantly the design performance.

The worst case optimization formulation [6] is given as

$$
\begin{aligned}
\min _{\rho} & : \max \left[c_{i}(\boldsymbol{\rho}), c_{e}(\boldsymbol{\rho}), c_{d}(\boldsymbol{\rho})\right] \\
\text { s.t. } & : \mathbf{K}\left(\left(\boldsymbol{\rho}, \eta_{i}\right)\right) \mathbf{u}_{i}=\mathbf{f}, \\
& : \mathbf{K}\left(\left(\boldsymbol{\rho}, \eta_{d}\right)\right) \mathbf{u}_{d}=\mathbf{f} \\
& : \mathbf{K}\left(\left(\boldsymbol{\rho}, \eta_{e}\right)\right) \mathbf{u}_{e}=\mathbf{f} \\
& : V\left(\boldsymbol{\rho}_{d}\left(\boldsymbol{\rho}, \eta_{d}\right)\right) \leq V^{*} \\
& :-1 \leq \boldsymbol{\rho} \leq 1
\end{aligned}
$$




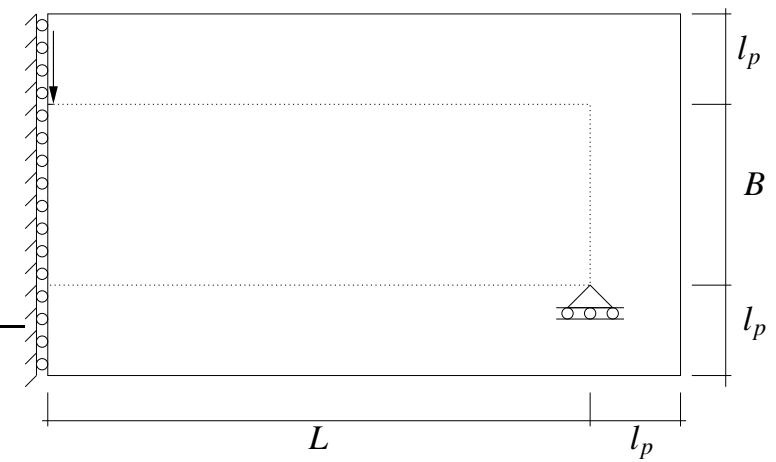

Figure 9: Design domain, boundary conditions and load for the MBB problem.

where $c_{i}, c_{e}$ and $c_{d}$ are computed for the intermediate, the most eroded and the most dilated cases. Compared to the original formulation Equation 1 the adopted worst case robust formulation requires the solution of three linear systems of equations corresponding to each of the considered design realizations. In practical implementations the optimization problem is reformulated to the so-called bound formulation [1]. The discrete max operator is replaced by an additional optimization variable and an additional constraint for each of the objective functions.

\subsection{MBB beam design}

The design domain with boundary conditions and the load for the so-called MBB (Messerschmitt - Bölkow - Blohm) beam problem are shown in Figure 9. In order to save computational cost only half of the problem is modeled. The ratio $L: B=3: 1$ is kept for all presented examples. The domain is padded in order to avoid an influence of the boundary conditions on the design in the filtering process. The padding $l_{p}$ distance depends on the filter parameters and the filter type. Concentrated unit force is applied at the upper right corner of the internal domain marked with dotted line.

For the examples presented in this section the padding length is set to $l_{p}=0.2 \mathrm{~B}$. The design field $\rho$ is set to zero in the padded part of the design domain. The domain is discretized using $140 \times 320$ elements. The initial $\beta$ is set to two and is multiplied by 1.2 every 50 iterations until beta is equal to $\beta=32$. The penalty is increased from 1 to 3 by multiplying it by a factor 1.2 every 50 iterations. The slow increase of $\beta$ and the penalty is required in order to provide more freedom to the optimizer in the early stages of the design process.

Non-robust designs with Gaussian filter defined by Equation 25 and different parameters are presented in Figure 10. The performance varies between the designs from 94.2 Nm to 96.1 Nm and improves slightly with increasing the central filter frequency. As expected, increasing the central filter frequency $\omega_{s}$ leads to more frequent appearance of solid elements and smaller void regions. It should be pointed out that the weights on the frequencies around the zero frequency are small but different from zero. Therefore, low frequency harmonics are allowed to be presented in the design. That is the main reason for the appearance of large void area in the upper right corners for all designs in Figure 10

Designs with the Gaussian filter defined by Equation 25 and zeroing the weights for all frequencies in a circle with radius $\omega_{s}-s$ and center $(0,0)$ are shown in Figure 11. This step removes completely the slowly varying harmonics from the design representation. Hence, the largest 

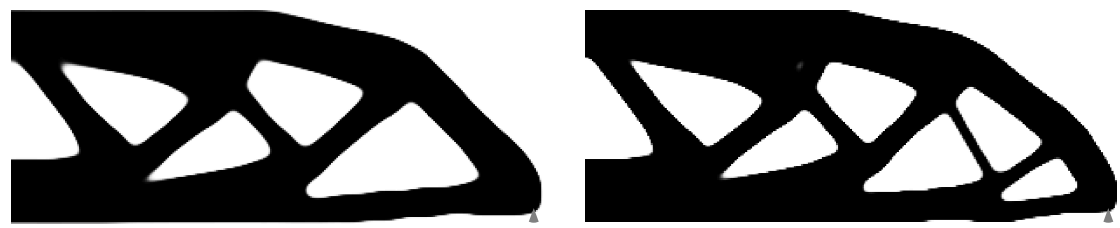

$\omega_{s}=1 B_{t}^{-1}, s=3 B_{t}^{-1}$

$\omega_{s}=3 B_{t}^{-1}, s=3 B_{t}^{-1}$
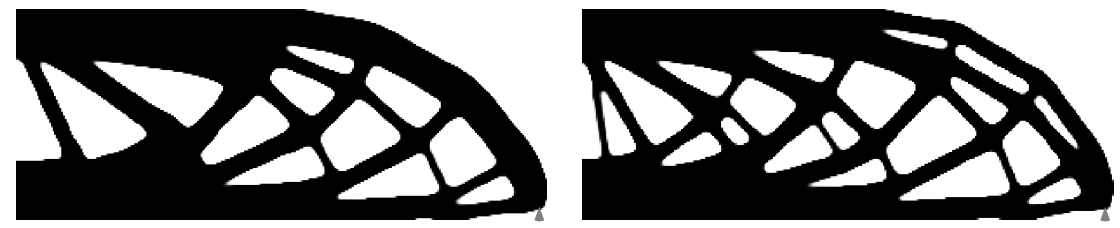

$\omega_{s}=5 B_{t}^{-1}, s=3 B_{t}^{-1}$

$\omega_{s}=6 B_{t}^{-1}, s=3 B_{t}^{-1}$

Figure 10: Non-robust MBB beam designs for different filter parameters where $B_{t}=B+2 l_{p}$.

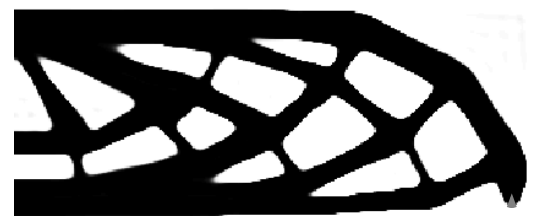

$\omega_{s}=5 B_{t}^{-1}, s=3 B_{t}^{-1}$

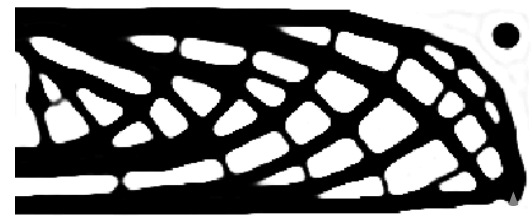

$\omega_{s}=6 B_{t}^{-1}, s=3 B_{t}^{-1}$

Figure 11: Non-robust MBB beam designs for different filter parameters and zero weight for all frequencies in a circle with radius $\omega_{s}-s$ and center equal to center of the frequency domain.

length between any two boundary points of the solid or the void regions has to be roughly smaller than the vertical dimension of the design domain divided by 2 for the first case with $\omega_{s}=5$ and by 3 for the second case $\omega_{s}=6$. This leads to the appearance of hanging solid inclusions which do not contribute to the design performance. They exist due to the imposed restrictions on the design space. Another expected effect due to the weight increase far from the center of the frequency domain is the appearance of thin elements in the design. As discussed earlier they can be suppressed by requiring robust performance with respect to geometric imperfections modeled using simple erosion and dilation realized by selecting different threshold projections. Such robust designs are shown in Figure 12. The thin elements are removed, however, in contrast to the non-robust design case free hanging solid regions appear in both problems. The appearance of hanging solid regions can be avoided by relaxing the filter to cover the entire frequency range as shown in Figure 10, by enforcing maximum length scale only on the solid phase of the design as demonstrated later in section 5, or by introducing additional constraint on the lower bound of the smallest eigenvalue for linear elastic structures [1].

The suggested band-pass filter projection scheme is recommended only for problems where the maximum size is defined loosely as a distribution in the frequency domain and is not crit- 


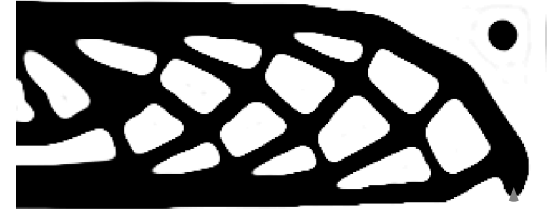

$\omega_{s}=5 B_{t}^{-1}, s=3 B_{t}^{-1}$

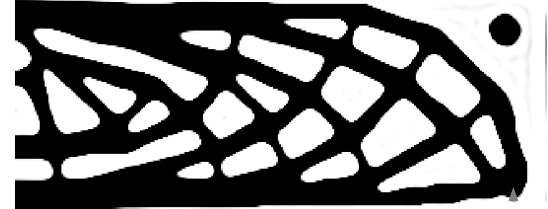

$\omega_{s}=6 B_{t}^{-1}, s=3 B_{t}^{-1}$

Figure 12: Robust MBB designs for different filter parameters and thresholds $\left[-0.015 M_{\rho_{s}} ; 0 ; 0.015 M_{\rho_{s}}\right]$.

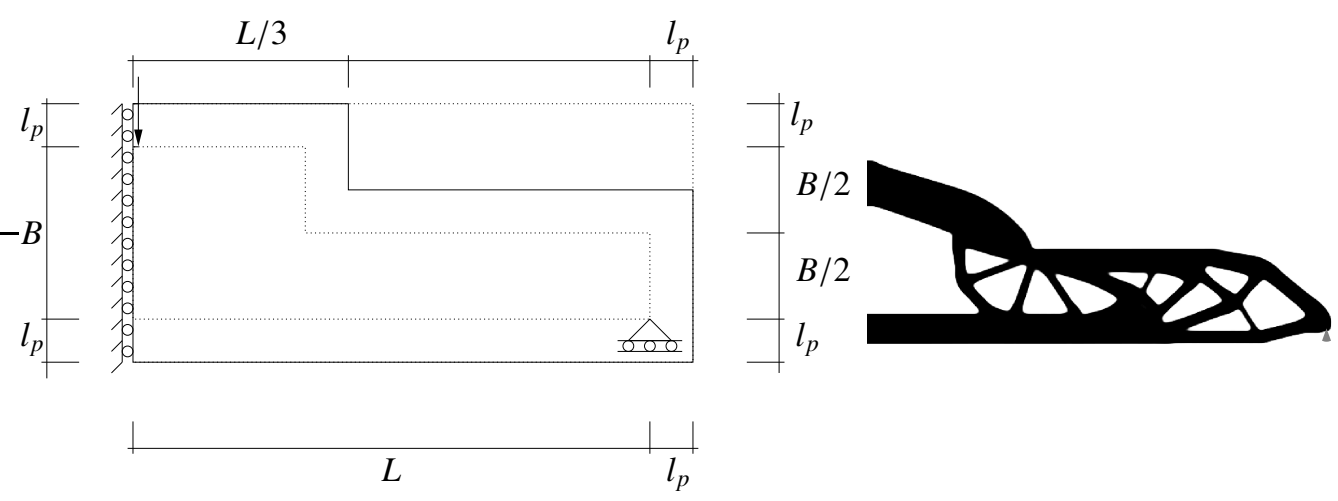

Figure 13: Design domain, boundary conditions and load for a non-rectangular MBB problem (left), and non-robust MBB design for filter parameters $\omega_{s}=7 B_{t}^{-1}, s=7 B_{t}^{-1}$.

ical for the final design realization and its performance. These are problems where repetitive patterns with specified frequency are desirable due to aesthetic or functional requirements, e.g., architectural designs, porosity in scaffold microstructures, designs of fill-in patterns in additive manufacturing. Alternative filtering-projections schemes for strict enforcement of maximum on the size of the design features and avoiding the appearance of artificial solid regions are presented in section 5 .

The applicability of the FFT filter for non-rectangular design domain is demonstrated on a minimum compliance optimization problem with boundary conditions and design domain shown in Figure 13. The padding $l_{p}$ is set to be $0.2 B$. The filter domain is extended to a rectangular domain in order to use FFT. The extension requires mapping from the actual design domain to the rectangular one. This complicates slightly the implementation, however, it gives a complete freedom to control the frequency content of the filtered design.

\section{Maximum length scale based on morphological operators}

The main limitation of the maximum length approach based on filtering is that the length scale cannot be stated in exact manner. It is loosely specified as a distribution in the frequency domain. Often in practice the maximum length scale is specified as a number which identifies the maximum dimension in a specified direction. Controlling such feature is difficult in the frequency domain which provides the motivation behind the approach presented in this section. 


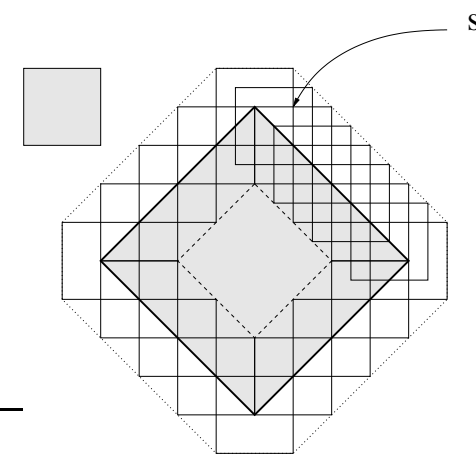

structuring element

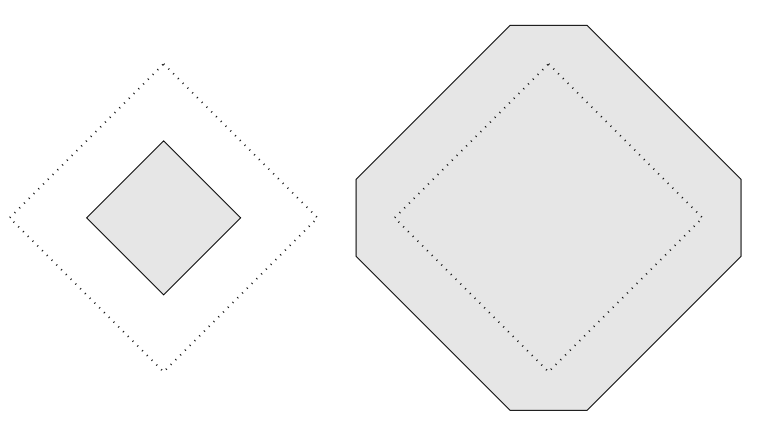

Figure 14: Example of erosion (middle) and dilation (right) operations applied on a rhomboid with square structural element shown in the left upper corner. The original shape is marked with dotted line in the transformed images.

The idea is built on standard operations utilized in image processing and developed over the last half century as a branch in mathematics known as mathematical morphology [16].

The two basic morphological operations, utilized here, are the erosion and the dilation operations. Initially the operations have been defined for binary $0 / 1$ images with the help of structuring element. The structuring element is a binary image usually defined on a sub-domain much smaller compared to the image domain. Its role is to select all points inside the sub-domain and either for dilation set them to one if a point inside the selection is one, or for erosion set them to zero if a point inside the selection is zero. Eroded or dilated versions of the binary image can be obtained by applying the above operations to all pixels. As mentioned in [25] the structuring element corresponds closely to the neighborhood operation for classical filtering in topology optimization. Examples of erosion and dilation operations are shown in Figure 14. As it can be seen the erosion removes a layer of material and dilation adds a layer of material along the perimeter of the solid region.

The applicability of morphological operators in topology optimization have been discussed in details in [25]. The logical operations for binary images are relaxed and replaced with continuous filter functions which mimic their behavior. A form of the dilation is utilized for imposing minimum length scale on the void or the solid phases of topology optimized designs in [4]. The idea is to apply a classical hat filter Equation 13 with a compact support on the design field and then project the result with Heaviside projection and threshold zero. Such an operation guarantees that the final projected density field is constructed by a union of solid circles, i.e., the minimum solid design feature is a circle with radius equal to the radius of the filter. The same idea can be applied on the void phase of the domain. Detailed discussion and comparison of the different approaches can be found in [6].

Here the morphological operators are applied for defining maximum length scale on the design. The length scale on the solid phase is defined by the parameter/parameters necessary to define the smallest structural element with specified shape for which the eroded design coincides with void design distribution everywhere in the design domain. Maximum length scale on the void is defined in a similar way by the parameter/parameters necessary to characterize the smallest structural element with specified shape for which the dilation coincides with solid design distribution everywhere in the design domain. The condition for the solid phase can be 
written as

$$
\int_{\Omega} \rho_{d e}(\mathbf{x}) \mathrm{d} \mathbf{x}=0
$$

and for the void phase

$$
\int_{\Omega}\left(1-\rho_{p d}(\mathbf{x})\right) \mathrm{d} \mathbf{x}=0
$$

where the fields $\rho_{d e}$ and $\rho_{p d}$ is obtained first by convolution with a filter function with compact support and then Heaviside projections. With the help of a relaxed Heaviside threshold projection [6] defined as

$$
H_{p}(\rho, \beta, \eta)=\frac{\tanh (\beta \eta)+\tanh (\beta(\rho-\eta))}{\tanh (\beta \eta)+\tanh (\beta(1-\eta))}
$$

and a filtered field $\rho_{d f}$

$$
\rho_{d f}(\mathbf{x})=\int_{\Omega} F(\mathbf{x}-\mathbf{y}) \rho_{d}(\mathbf{y}) \mathrm{d} \mathbf{y}
$$

the eroded field for Equation 28 is defined as

$$
\rho_{d e}(\mathbf{x})=H_{p}\left(\rho_{d f}(\mathbf{x}), \beta, \eta=1\right)
$$

and the dilated field for Equation 29 is defined as

$$
\rho_{p d}(\mathbf{x})=H_{p}\left(\rho_{d f}(\mathbf{x}), \beta, \eta=0\right)
$$

The modified discrete topology optimization problem with maximum length scale on both phases is defined as

$$
\begin{gathered}
\min _{\rho}: c(\boldsymbol{\rho}), \\
\text { s.t. }: \mathbf{K}\left(\boldsymbol{\rho}_{d}(\boldsymbol{\rho})\right) \mathbf{u}=\mathbf{f}, \\
V\left(\boldsymbol{\rho}_{d}(\boldsymbol{\rho})\right) \leq V^{*}, \\
\sum \boldsymbol{\rho}_{d e, e} \leq \varepsilon_{s} \\
\sum\left(1-\boldsymbol{\rho}_{d d, e}\right) \leq \varepsilon_{v} \\
\\
0 \leq \boldsymbol{\rho} \leq 1
\end{gathered}
$$

where $\boldsymbol{\rho}_{d}=H_{p}\left(\boldsymbol{\rho}_{s}, \beta, \eta\right)$ is relaxed Heaviside projection of the filtered field vector $\boldsymbol{\rho}_{s}$ obtained by filtering of the design field vector $\rho$. The filter can be constructed by any of the filtering techniques discussed in section 3 . The design field is this case is bounded between zero and one. The first sum corresponds to the integral defined by Equation 28 and the second one to the integral defined by Equation 29 Hence, the first sum enforces maximum length scale on the solid phase and the second sum maximum length scale on the void phase. The max-length scale constraints can be relaxed by setting the values $\varepsilon_{s}$ and $\varepsilon_{v}$ to small positive numbers larger than zero. Enforcing both constraints results in a maximum length scale on both phases. Removing one of them results in maximum length scale on one of the phases which widens the applicability range compared to the approach presented in section 4 where maximum length scale is imposed on both phases. The material properties for each element are obtained by the SIMP interpolation scheme. 

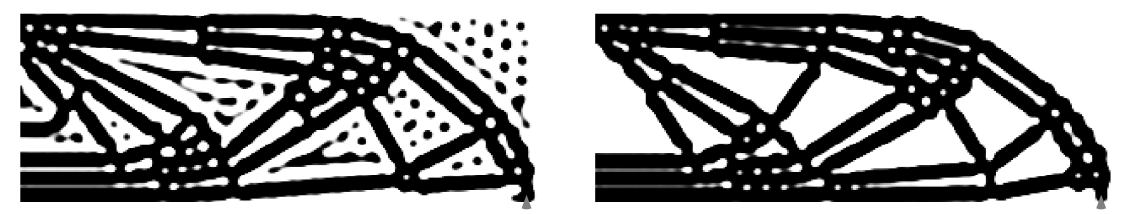

Figure 15: MBB beam designs with maximum length scale imposed on both phases (left) and only on the solid phase (right).

\subsection{MBB beam design}

The first example is optimized design of MBB beam with design domain and boundary conditions shown in Figure 9. The padding length is set to $l_{p}=2 r$ where $r=0.056 B$. The internal part of the design domain marked with dotted line is discretized using $300 \times 100$ elements. The designs are obtained using $\beta$ continuation where $\beta=2$ in the beginning and is multiplied by 2 every 50 iterations. The final value is $\beta=32$. Non-robust designs with maximum length scale imposed on both phases and only on the solid phase are shown in Figure 15 Imposing maximum length scale on both phases results in solid elements which do not contribute to the design performance. These artifacts, similar to case with band-pass filters, appear only due to the imposed constraints. Removing the requirement for maximum length scale on the void regions alleviates the issue of hanging solid features. This demonstrates the flexibility of the additional constraints approach compared to the band pass filter - projection methodology presented earlier in this article.

Close study of the results in Figure 15 reveals that even though a relatively large projection parameter is utilized in the final design the projected density field possesses gray regions, which require post processing. These gray regions are removed using the recently proposed methodology [34] for imposing minimum length scale using only additional geometric constraints. The results are shown in Figure 16. The idea is to require that the filtered field $\rho_{f}$ is above a threshold $\eta_{e}$ for all points $\mathbf{x}=\left\{\mathbf{y} \in \Omega \mid \rho_{d}(\mathbf{y})=1\right.$, and $\left.\nabla \rho_{f}=0\right\}$. The above condition imposes length scale on the solid phase only. Additional condition where the filtered field $\rho_{f}$ is required to be below a threshold $\eta_{d}$ for all points $\mathbf{x}=\left\{\mathbf{y} \in \Omega \mid \rho_{d}(\mathbf{y})=0\right.$, and $\left.\nabla \rho_{f}=0\right\}$, imposes minimum length scale on the void phase. The initial designs for both optimization runs are the final designs shown in Figure 15, and the thresholds are selected to be $\eta_{d}=0.35$ and $\eta_{e}=0.65$. For more implementation details the interested readers are referred to [34]. It should be pointed out that in this case the designs response is not required to be robust with respect to geometric variations in the design.

The optimized designs with maximum length scale posses large number of hollow circles close to the elements connections. These are natural outcome of the imposed maximum length scale constraints. Their appearance can be controlled completely by either increasing the length scale of the void regions or by decreasing the volume fraction of the solid phase. The length scale in the void regions can be increased by moving the dilated realization threshold closer to zero, and/or increasing the filter radius [6]. The effect can be seen in Figure 18 where the length scale on the void phase is increased by doubling the filter radius and moving the dilated threshold to 0.23 . The threshold is selected to match the minimum length scale imposed on the solid phase for the designs presented in Figure 17 and Figure 18. The imposed maximum/minimum length scale constraints require rounding of all angles, i.e., all elements connecting to a common node have to be tangent to a circle. The restriction leads to wavy behavior of the elements around the connections. The reduction of the solid material, on the other hand, forces the optimization 

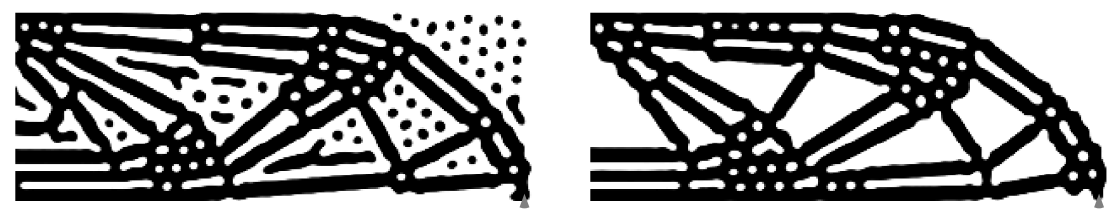

Figure 16: MBB designs with minimum and maximum length scale imposed on both phases (left) and maximum length scale only on the solid phase (right).

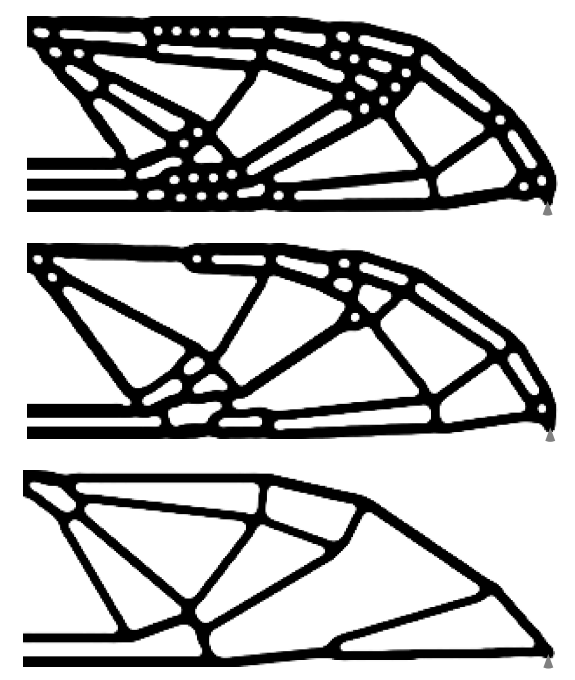

Figure 17: MBB designs with maximum length scale imposed only on the solid phase and minimum length scale imposed on both phases and volume fractions of $40 \%, 30 \%$, and $20 \%$.

to keep the main structural elements and removes the less critical details, thus, reducing the design complexity. Due to the smaller minimum length scale imposed on the void phase the wavy behavior of elements around the connections is not strongly pronounced. The effect is demonstrated in Figure 17] for volume fractions $40 \%, 30 \%$, and $20 \%$. The designs are obtained using robust formulation [6] with initial design shown in Figure 16 right. The maximum length scale on the solid phase for the designs presented in Figure 17] and Figure 18] is the same.

\subsection{Compliant mechanism design with maximum length scale}

The second example is the design of compliant mechanism with design domain and boundary conditions shown in Figure 19 The optimization parameters are the same as for the MBB design in subsection 5.1. The input spring has unit stiffness and the output spring is $10^{3}$ softer than the input one. A unit force $f_{\text {in }}$ is applied on the upper left corner of the design domain and the objective is to maximize displacement $u_{\text {out }}$. The response is required to be robust with respect to uniform erosion and dilation within the threshold interval $[0.35,0.65]$. The optimization is performed using the min / max formulation, e.g. Equation 27, with three thresholds $\eta_{e}=0.65$, $\eta_{i}=0.5$, and $\eta_{d}=0.35$. Detailed study of the formulation can be found in [6].

The optimized results of the compliant mechanism for volume fractions of $35 \%$ and $20 \%$ are shown in Figure 20 The first design is obtained with maximum length scale imposed on 


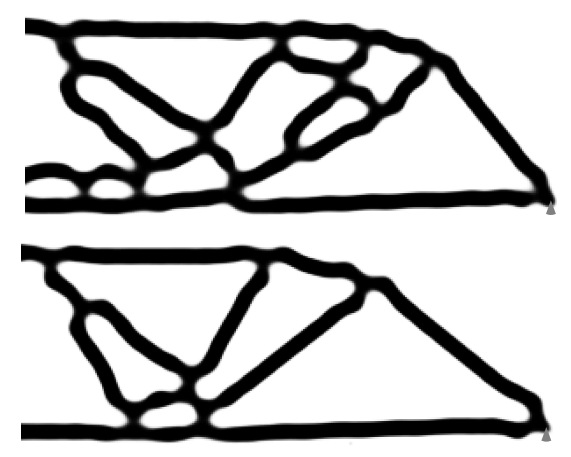

Figure 18: MBB designs with maximum length scale imposed only on the solid phase and minimum length scale imposed on both phases and volume fractions of $30 \%$ and $20 \%$. The length scale of the void phase is increased by doubling the filter radius used in Figure 17 and by using dilated realization for threshold of 0.23 .

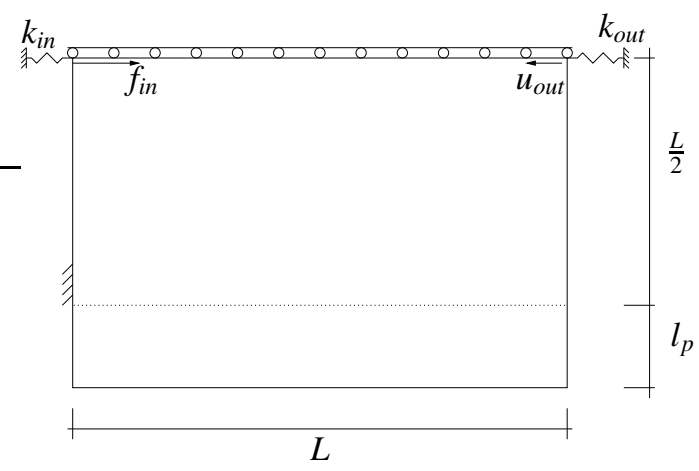

Figure 19: Compliant mechanism - design domain and boundary conditions. 

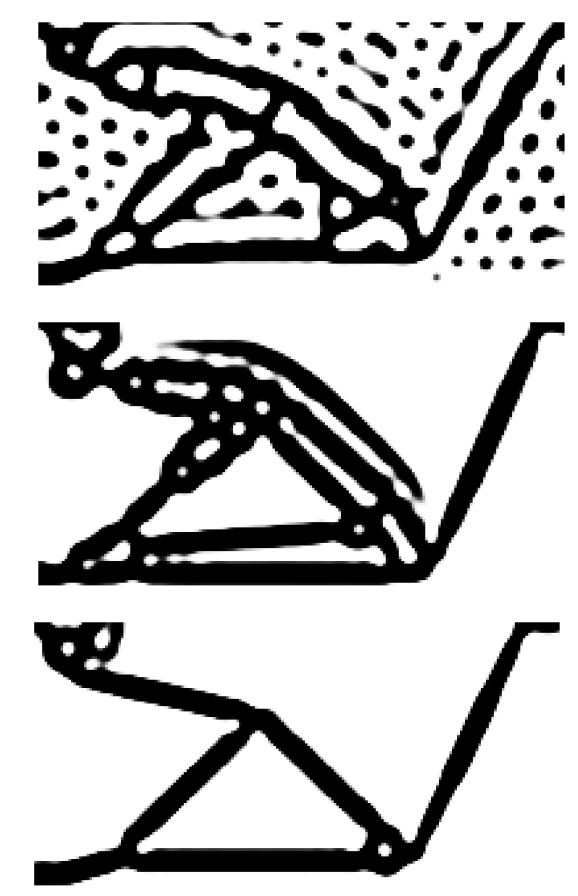

Figure 20: Compliant mechanism design with maximum length scale imposed on both phases, and only on the solid phase. The first two designs are obtained with volume constraint $35 \%$ of the total volume and the last design is obtained with volume constraint $20 \%$ of the total volume of the design domain.

both phases. Isolated solid regions are distributed in the design domain in order to fulfill the condition for imposing maximum length scale on the void phase. Requiring maximum length scale only on the solid regions removes the hanging solid features. However, close inspection reveals that a large solid element is connected to the main structure by weak gray connections. This, can be attributed to the highly non-convex nature of the optimization problem. Furthermore it indicates that the volume constraint becomes inactive. Due to the continuation scheme the design topology can get locked in the early stages of the optimization, and later when the length scale constraints become active the optimizer cannot utilize the excess material. Decreasing the material volume to $20 \%$ removes the weakly connected elements. Another well known feature is that the requirement for robustness of the mechanism performance alleviates the single node hinge problem [6] and imposes minimum length scale for the parts of the structure which actively contribute to the design performance, i.e., all critical regions around the flexible hinges posses minimum length scale. However, regions/elements which cannot provide significant changes in the design performance might not exist in all design realizations and hence length scale for them is not defined. As the performance of the design is not affected significantly by modifying these elements, the design is manufacturable within the allowed manufacturing imperfections.

The convergence history is shown in Figure 21. The jumps in the performance for the different realizations correspond to increase of the parameter $\beta$. As discussed in [6], the eroded and the dilated realizations show equal performance for the optimized topology. 


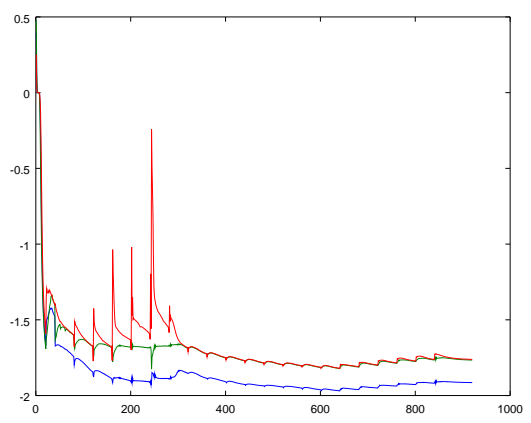

Figure 21: Convergence history - compliant mechanism design. Eroded, intermediate and dilated realizations are shown with red, blue and green colors, respectively.

\section{Conclusions}

An alternative for filtering based on Fourier transform, and two new alternatives for imposing maximum length scale in density based topology optimization are presented in details. The methods eliminate the need of large number of constraints and an implementation requires slight modification of the existing topology optimization formulations. Their limitations and advantages are demonstrated in optimizations of linear elastic designs. The methods are general and applications to other optimization problems will be demonstrated in future research. The proposed maximum length scale alternatives can be combined with most methods for imposing minimum length scale providing the designers with complete control over the design process and resulting in manufacturable robust black and white designs without any post processing.

\section{Acknowledgments}

The first author is funded by Villum Fonden through the NextTop project, as well as the EU FP7-MC-IAPP programme LaScISO. The second author is funded by an ERC Starting Grant through INNODYN.

\section{References}

[1] M. P. Bendsøe, O. Sigmund, Topology Optimization - Theory, Methods and Applications, Springer Verlag, Berlin Heidelberg, 2003.

[2] B. Bourdin, Filters in topology optimization, International Journal for Numerical Methods in Engineering 50 (2001) $2143-2158$

[3] T. E. Bruns, D. A. Tortorelli, Topology optimization of non-linear elastic structures and compliant mechanisms, Computer Methods in Applied Mechanics and Engineering 190 (2001) 3443-3459.

[4] J. Guest, J. Prevost, T. Belytschko, Achieving minimum length scale in topology optimization using nodal design variables and projection functions, International Journal for Numerical Methods in Engineering 61 (2) (2004) 238254. doi: $\{10.1002 / \mathrm{nmc} .1064\}$

[5] E. Andreassen, B. S. Lazarov, O. Sigmund, Design of manufacturable 3d extremal elastic microstructure, Mechanics of Materials 69 (1) (2014) $1-10$. doi:10.1016/j.mechmat.2013.09.018

[6] F. Wang, B. Lazarov, O. Sigmund, On projection methods, convergence and robust formulations in topology optimization, Structural and Multidisciplinary Optimization 43 (6) (2011) 767-784 doi:10.1007/s00158-010-0602-y 
[7] J. Guest, Imposing maximum length scale in topology optimization, Structural and Multidisciplinary Optimization 37 (2009) 463-473, 10.1007/s00158-008-0250-7.

[8] M. Jansen, G. Lombaert, M. Schevenels, O. Sigmund, Topology optimization of fail-safe structures using a simplified local damage model, Structural and Multidisciplinary Optimization 49 (4) (2014) 657-666. doi:10.1007/s00158-013-1001-y

[9] S.-H. Ha, J. Guest, Optimizing inclusion shapes and patterns in periodic materials using discrete object projection, Structural and Multidisciplinary Optimization 50 (1) (2014) 65-80. doi:10.1007/s00158-013-1026-2

[10] J. K. Guest, Optimizing the layout of discrete objects in structures and materials: A projection-based topology optimization approach, Computer Methods in Applied Mechanics and Engineering 283 (0) (2015) $330-351$. doi:http://dx.doi.org/10.1016/j.cma.2014.09.006

[11] G. Michailidis, Manufacturing Constraints and Multi-Phase Shape and Topology Optimization via a Level-Set Method. $\mathrm{Ph} . \mathrm{D}$. thesis, Ecole Polytechnique X (Jan 2014).

URL https://pastel.archives-ouvertes.fr/pastel-00937306

[12] G. Allaire, F. Jouve, G. Michailidis, Thickness control in structural optimization via a level set method (Apr. 2014). URL https://hal.archives-ouvertes.fr/hal-00985000

[13] X. Guo, W. Zhang, W. Zhong, Explicit feature control in structural topology optimization via level set method, Computer Methods in Applied Mechanics and Engineering 272 (0) (2014) 354 - 378. doi:10.1016/j.cma.2014.01.010

[14] W. Zhang, W. Zhong, X. Guo, An explicit length scale control approach in simp-based topology optimization, Computer Methods in Applied Mechanics and Engineering 282 (0) (2014) 71 - 86. doi:10.1016/j.cma.2014.08.027

[15] T. S. Kim, J. E. Kim, J. H. Jeong, Y. Y. Kim, Filtering technique to control member size in topology design optimization, KSME International Journal 18 (2) (2004) 253-261. doi:10.1007/BF03184735

[16] L. Najman, H. Talbot, Mathematical Morphology: From Theory to Applications, John Wiley \& Sons, Inc., 2013. doi:10.1002/9781118600788

[17] M. P. Bendsøe, O. Sigmund, Material interpolation schemes in topology optimization, Archive of Applied Mechanics 69 (1999) 635-654.

[18] A. Diaz, O. Sigmund, Checkerboard patterns in layout optimization, Structural and Multidisciplinary Optimization 10 (1995) 40-45

[19] C. S. Jog, R. B. Haber, Stability of finite element models for distributed-parameter optimization and topology design, Computer Methods In Applied Mechanics And Engineering 130 (3-4) (1996) 203-226.

[20] B. S. Lazarov, O. Sigmund, Filters in topology optimization based on helmholtz-type differential equations, International Journal for Numerical Methods in Engineering 86 (6) (2011) 765-781. doi:10.1002/nme.3072

[21] D. E. Newland, An introduction to random vibrations, spectral and wavelet analysis, Longman Scientific and Technical, 1993

[22] E. Andreassen, A. Clausen, M. Schevenels, B. Lazarov, O. Sigmund, Efficient topology optimization in matlab using 88 lines of code, Structural and Multidisciplinary Optimization 43 (2011) 1-16. doi : 10.1007/s00158-010-0594-7

[23] K. Svanberg, The method of moving asymptotes - a new method for structural optimization, International Journal for Numerical Methods in Engineering 24 (1987) 359-373.

[24] Y. Elesin, B. Lazarov, J. Jensen, O. Sigmund, Design of robust and efficient photonic switches using topology optimization, Photonics and Nanostructures - Fundamentals and Applications 10 (1) (2012) 153 - 165 doi:10.1016/j.photonics.2011.10.003

[25] O. Sigmund, Morphology-based black and white filters for topology optimization, Structural and Multidisciplinary Optimization 33 (2007) 401-424. doi : 10.1007/s00158-006-0087-x

[26] S. Xu, Y. Cai, G. Cheng, Volume preserving nonlinear density filter based on heaviside functions, Structural and Multidisciplinary Optimization 41 (2010) 1615-1488.

[27] A. Kawamoto, T. Matsumori, S. Yamasaki, T. Nomura, T. Kondoh, S. Nishiwaki, Heaviside projection based topology optimization by a pde-filtered scalar function, Structural and Multidisciplinary Optimization 44 (2011) 19-24. doi:10.1007/s00158-010-0562-2

[28] O. Sigmund, Manufacturing tolerant topology optimization, Acta Mechanica Sinica 25 (2009) 227-239.

[29] B. S. Lazarov, M. Schevenels, O. Sigmund, Robust design of large-displacement compliant mechanisms, Mechanical Sciences 2 (2) (2011) 175-182. doi:10.5194/ms-2-175-2011

[30] M. Schevenels, B. Lazarov, O. Sigmund, Robust topology optimization accounting for spatially varying manufacturing errors, Computer Methods in Applied Mechanics and Engineering 200 (49-52) (2011) 3613 - 3627. doi:10.1016/j.cma.2011.08.006

[31] B. S. Lazarov, M. Schevenels, O. Sigmund, Topology optimization considering material and geometric uncertainties using stochastic collocation methods, Structural and Multidisciplinary Optimization 46 (2012) 597-612. 
doi:10.1007/s00158-012-0791-7

[32] M. Jansen, B. Lazarov, M. Schevenels, O. Sigmund, On the similarities between micro/nano lithography and topology optimization projection methods, Structural and Multidisciplinary Optimization 48 (4) (2013) 717-730. doi:10.1007/s00158-013-0941-6

[33] M. Zhou, B. S. Lazarov, O. Sigmund, Topology optimization for optical projection lithography with manufacturing uncertainties, Applied Optics 53 (12) (2014) 2720-2729. doi:10.1364/A0.53.002720

[34] M. Zhou, B. S. Lazarov, F. Wang, O. Sigmund, Minimum length scale in topology optimization by geometric constraints, Computer Methods in Applied Mechanics and Engineering 293 (0) (2015) 266 - 282. doi:10.1016/j.cma.2015.05.003 